\title{
Questes
}

Revue pluridisciplinaire d'études médiévales

16 | 2009

Secret, public, privé

\section{Le secret dans les représentations romanesques de la pratique épistolaire}

\section{Fanny Oudin}

\section{(2) OpenEdition}

\section{Journals}

\section{Édition électronique}

URL : http://journals.openedition.org/questes/724

DOI : 10.4000 /questes.724

ISSN : 2109-9472

\section{Éditeur}

Les Amis de Questes

\section{Édition imprimée}

Date de publication : 15 avril 2009

Pagination : 25-38

ISSN : 2102-7188

\section{Référence électronique}

Fanny Oudin, «Le secret dans les représentations romanesques de la pratique épistolaire », Questes

[En ligne], 16 | 2009, mis en ligne le 01 janvier 2014, consulté le 01 mai 2019. URL : http:// journals.openedition.org/questes/724; DOI : 10.4000/questes.724 


\title{
Le secret dans les représentations romanesques de la pratique épistolaire
}

\author{
Fanny OUDIN
}

Le roman médiéval ${ }^{1}$ aime s'attarder sur les difficultés matérielles et les échecs de la correspondance. La forme narrative la plus riche du message est celle de la lettre déviée et faussée, qui ne parvient pas à remplir sa fonction et n'atteint pas le but qu'elle s'était proposé. Mais avant même d'être échangées, les lettres sont d'abord lues par des intrus, à qui elles n'étaient pas destinées. Plus que le geste d'inversion du faussaire, si transparent que le texte authentique de la lettre réapparaît toujours en palimpseste à travers le texte de la fausse lettre ${ }^{2}$, c'est l'interception qui, à l'origine de tous les déboires de la communication épistolaire, attire l'attention ${ }^{3}$. Le premier thème à apparaître

\footnotetext{
${ }^{1}$ Le corpus sur lequel s'appuie cet exposé est volontairement restreint aux romans en vers des $\mathrm{XII}^{\mathrm{e}}$ et XIII ${ }^{\mathrm{e}}$ siècles, à l'exclusion des romans arthuriens et des romans d'Antiquité. Sont pris en compte en particulier Partonopeus de Blois, Eracle de Gautier d'Arras, Protheselaüs de Hue de Rotelande, Galeran de Bretagne de Renaut, Le Roman de Silence d'Heldris de Cornouailles, La Manekine de Philippe de Remi, Le Roman du comte d'Anjou de Jean Maillart, Le Roman du châtelain de Couci et de la dame de Fayel de Jakemes.

2 Tous les personnages qui ont affaire à de fausses lettres, du lecteur de Protheselaüs jusqu'au roi d'Écosse, rétablissent instinctivement le texte original, en opposant ce qu'ils attendaient de la missive, et qui était son contenu authentique, et ce que leur présente la fausse lettre. À la technique d'inversion systématique de l'énoncé qui est celle de faussaires comme Brutus, qui tourne systématiquement l'amitié en haine, correspond donc un mouvement inverse au moment de la lecture. Celui-ci n'émerge cependant que rarement à la conscience : seul le comte de Clermont effectue le passage du contraste entre son attente et la lettre qu'il tient à l'hypothèse d'une falsification.

${ }^{3}$ Il est de ce point de vue étrange que Jacques MERCERON, dans Le Message et sa fiction. La communication par messager dans la littérature française des XII et XIII siècles, Berkeley, University of California Press, 1998, ne relève pas l'interception comme un motif de «médiation problématique » du message écrit dans le chapitre qu'il consacre à ce problème, puisqu'il existe des scènes d'interruption de la communication qui n'aboutissent pas nécessairement à une falsification proprement dite, comme dans le Roman du châtelain de Couci.
} 
dans ces scènes est donc celui de la lettre violée, qui pose le problème de l'importance du secret au sein de l'échange épistolaire. À travers ce motif de la communication secrète, qui fait rimer « scellé » avec « celé », est interrogée la nature des liens interpersonnels qui se nouent dans la relation épistolaire, mais aussi les rapports que la correspondance entretient avec les sphères du public et du privé, ainsi que les connexions dynamiques qu'elle établit entre les deux. Dans l'ouvrage qu'il a dirigé sur Les usages de la lettre au XIX siècle, Roger Chartier insiste en effet à plusieurs reprises sur le caractère privilégié du geste épistolaire pour saisir l'équilibre établi entre le moi et les autres, que ceux-ci soient envisagés individuellement ou collectivement, comme personnes ou comme groupe social. Pour lui, à la fois libre et codifiée, alliant l'intime au public aussi bien que le secret à la sociabilité, «la lettre, mieux qu'aucune autre expression, associe le lien social et la subjectivité ${ }^{4}$. Dans les romans médiévaux, c'est visiblement à partir du motif de la rupture du secret que se formulent de façon privilégiée ces relations, sous la figure de l'intrusion d'un tiers, d'une altérité radicale et indésirable, au sein d'une relation interpersonnelle singulière.

\section{Les cercles concentriques du secret : une communication privée}

De façon remarquable, les lettres transmises d'un personnage à l'autre sont systématiquement, et quel que soit le contexte, des lettres scellées et fermées. Aucun exemple de lettre patente ${ }^{5}$ n'est mentionné : lorsqu'un personnage, comme l'empereur Laïs d'Eracle, veut faire circuler une

\footnotetext{
${ }^{4}$ CHARTIER, Roger (dir.), La Correspondance. Les usages de la lettre au XIXe siècle, Paris, Fayard, 1991, p. 10.

${ }^{5}$ Ce sont des lettres ouvertes, dont la singularité tient justement à ce que, même si le sceau y est apposé et y a valeur d'autorité, elles ne sont pas repliées sur elles-mêmes et leur contenu reste visible de tous.
} 
information ou un ordre de façon ouverte, il utilise la proclamation orale en faisant « son ban crier ${ }^{6}$. La rime entre $« \operatorname{mox} »$ et $«$ enclox », récurrente dans la présentation des lettres, met en exergue cette fermeture sur elle-même de la lettre: celle-ci privatise l'information transmise en la liant à la relation interpersonnelle sur laquelle repose sa transmission. La périphrase par laquelle Isabelle désigne les lettres lorsqu'elle demande au messager du châtelain de l'attendre est par exemple révélatrice :

«Et si t'aporterai deus mos

En un poi de parcemin clos,

Que tu reporteras celui

Qui ces lettres m'envoia hui. » ${ }^{7}$

Le contenu du message semble de valeur plutôt négligeable ${ }^{8}$ alors que l'attention se concentre sur la clôture et la personnalisation du discours, qui vont de pair : l'adresse et le sceau sont liés dans la définition même du geste épistolaire. La discrétion d'Isabelle, sa tendance à minimiser l'importance réelle du message transmis se justifient par la nécessité du secret inhérent à l'amour courtois. Par là, la communication « seelee » tend à se transformer en communication «celee», car le messager doit toujours s'arranger pour

${ }^{6}$ Gautier D’Arras, Eracle, éd. Guy Raynaud de Lage, Paris, CFMA, 1976, v. 728 et v. 1273.

7 JAKEMES, Le Roman du Castelain de Couci et de la Dame de Fayel, éd. Maurice Delbouille, Paris, SATF, 1936, v. 3123-3126.

${ }^{8}$ Cette rime sur «enclos » est aussi une rime caractéristique de la définition poétique des textes (il suffit de songer à la célèbre rime du Roman de la Rose, antérieur au roman de Jakemes). De ce point de vue, la fermeture de la lettre qui se replie sur des «mots », et non sur des idées, évoque le mouvement des fleurs qui encerclent le salut de Guillaume : ce geste de clôture correspondrait donc également à une perfection poétique de l'épître close sur elle-même, autonome dans son devenir textuel. Mais cette autonomie est mise en balance par l'adresse, qui la suit immédiatement et qui, destinant la missive à une personne donnée, l'ouvre de nouveau sur une subjectivité : le texte épistolaire ne parvient pas à se détacher totalement de la relation dans laquelle il est pris, et ne revêt véritablement tout son sens que par rapport à un destinataire particulier, dont il dépend. C'est tout le paradoxe de la lettre d'être tendue entre ces deux statuts, et c'est ce qui fait la particularité du secret qu'elle met en jeu. 
remettre sa missive au destinataire «celeement»: l'emploi de cet adverbe pour qualifier le processus de la communication épistolaire est des plus fréquents dans tous les romans.

Par contraste, la mise en scène de la rupture de ce secret épistolaire est des plus violentes. Dans Galeran par exemple, le manque de discrétion du porteur des missives donne lieu à une intrusion de l'abbesse Hermine dont la brutalité est renforcée par celle de ses gestes et de ses paroles $^{9}$ : la lettre est non seulement déchirée mais foulée aux pieds, le messager chassé et Frêne blessée $^{10}$ et insultée devant lui. La même violence verbale se retrouve dans les propos menaçants adressés à Gobiers par le seigneur du Fayel pour lui extorquer la missive dont il est porteur, mais les gestes qui président à la transgression du secret épistolaire sont plus mesurés ${ }^{11}$. C'est alors par une série d'effractions successives qu'est rendue palpable la violation: pour atteindre la lettre convoitée, tous ceux qui cherchent à s'en emparer doivent d'abord venir à bout du messager puis, systématiquement, ouvrir la boîte où est abritée la lettre en forçant la serrure ${ }^{12}$. Dans ce roman, la lettre est en outre

\footnotetext{
${ }^{9}$ RENAUT, Galeran de Bretagne, v. 3632 sqq., éd. par Lucien Foulet, Paris, CFMA, 1925.

${ }^{10}$ Légèrement il est vrai, mais nous sommes dans un roman courtois. Que Renaut prenne la peine de signaler, non sans humour peut-être, cet ongle retourné témoigne d'une insistance sur la furie qui emporte chacun des gestes de l'abbesse: elle ne se maîtrise plus, et ce manque de maîtrise chez une personne censée faire preuve par excellence de mesure et de dignité est un indice fort de la violence de l'interruption de la correspondance.

11 JAKEMES, Le Roman du Castelain de Couci et de la Dame de Fayel, v. 7895-8016.

12 Je suis sur ce point en désaccord avec Danielle QUERUEL qui, dans son article « Lettres d'amour et art épistolaire dans le Roman du châtelain de Couci et de la dame de Fayel », dans Plaist vos oür bone cançon vaillant? Mélanges de langue et de littérature médiévales offerts à François Suard, textes réunis par Dominique BOUTET, Marie-Madeleine CAStellani, Françoise FerRAND et Aimé Petit, Lille, t. II, 1999, p. 759-771, affirme que « le mari prend alors la précaution de ne pas briser la serrure du coffret, ni le sceau de la lettre, ouvre la lettre avec soin, la lit et la referme sans laisser de trace ». Les vers qu'elle cite elle-même à la suite de ce jugement me semblent au contraire souligner non la douceur mais la violence de l'effraction puisque le seigneur de Fayel «Clef ne sierre n'a demandé, / Mais a force l'a deffremé. » (v. 8001-8002).
} 
recouverte des tresses de la dame et du cœur du châtelain, que le seigneur du Fayel doit donc déplacer pour y accéder. Or il s'agit de morceaux du corps des deux interlocuteurs, qui les représentent par métonymie : la transgression d'une relation intime n'en est que plus palpable, puisque c'est dans leur chair que les correspondants sont atteints.

La même succession d'obstacles à l'infraction crée dans Protheselaüs ${ }^{13}$ un jeu de cercles concentriques qui restreignent progressivement l'espace jusqu'à ce que soit atteinte la lettre elle-même. Elle apparaît ainsi comme le point le plus privé de cet espace. Pour l'atteindre, Brutus doit en effet assommer la sentinelle du navire, puis forcer la porte de la cabine, fouiller les malles, en extraire la boîte du messager, et ouvrir la lettre sans briser le cachet de cire pour pouvoir le réutiliser. Outre le garde et le sceau, ce parcours implique donc que pas moins de trois serrures soient forcées successivement. L'insistance particulière sur la fouille de la cabine, qui prend des allures de cambriolage avec l'accumulation négative de tout ce que les intrus ne cherchent pas, donne tout son relief à cette série d'effractions successives qui aboutissent à une concentration sur la lettre, présentée comme un refuge ultime de l'intimité. Le secret épistolaire est donc bien ici un secret dont l'évaluation est positive, un secret violé, et non un secret dévoilé, qui aurait fait l'objet d'une dissimulation indue.

\section{Secrets privés, effractions politiques ?}

L'effraction a pour fonction de souligner le caractère personnel et intime de tout courrier, qui n'est pas destiné à être lu par n'importe qui, contrairement à la rumeur qui vise la diffusion la plus large possible. Mais une

\footnotetext{
13 Hue de Rotelande, Protheselaüs, éd. Anthony J. Holden, London, Anglo-Normand
} Text Society, 3 vol., 1991, v. 551-592. 
ambiguïté apparaît quant à la nature et la définition précise de cette intimité qui demande à être préservée : est-ce au caractère secret à proprement parler ou au caractère privé de la correspondance que l'intrusion de tiers porte atteinte ? Secret et privé, en effet, ne se confondent pas : une relation privée, par exemple, peut très bien n'être pas tenue secrète.

A priori pourtant, secret et privé se rencontrent dans une commune opposition au domaine public: le secret renfermerait tout ce qui n'est pas ostensible et manifeste, ce qui échappe à la perception du public, tandis que le privé, s'opposant plus particulièrement à la définition qui fait coïncider public et politique, désignerait ce qui fait l'objet d'une appropriation et d'une forme de pouvoir particulières, par opposition au bien commun ${ }^{14}$. Dans cette perspective, les lettres analysées seraient à la fois secrètes et privées, reposant sur une relation intime, et non politique, et sur une dissimulation de l'information qui la soustrait au regard d'autrui. Ainsi, la réaction de l'abbesse Hermine se justifie par la réintroduction de considérations politiques, relatives aux rangs respectifs des interlocuteurs dans la société féodale, dans une relation à la fois privée et cachée. De même, le seigneur du Fayel se réclame de pouvoirs familiaux ${ }^{15}$ pour briser et le secret de la correspondance et la relation personnelle qu'elle actualisait. De fait, la majorité des secrets épistolaires, même s’ils ne se restreignent pas à de simples secrets amoureux, relèvent du domaine privé ${ }^{16}$ : privé et secret coïncideraient donc bien, et

${ }^{14}$ Duby, Georges (dir.), Histoire de la vie privée, Tome II, De l'Europe féodale à la Renaissance, Paris, Seuil, 1999.

${ }^{15}$ Le cercle familial relève certes d'un espace privé, mais qui reste sociabilisé et régi par un jeu de pouvoirs, alors que la relation amoureuse qui est visée correspond elle à la sphère de l'intimité individuelle, qui s'y emboîte : le type de lien social dont joue le seigneur du Fayel est donc bien plus public, comparativement, que celui auquel il s'en prend.

${ }^{16}$ L'annonce de naissances, par exemple, qui constitue l'autre grand thème des lettres avec l'amour, est bien à l'origine une nouvelle strictement privée. 
l'intrusion d'autrui correspondrait à la fois à une rupture du secret et à l'introduction de visées politiques sur une relation privée.

Cette coïncidence apparente ne résout cependant pas le problème de la nature de la personnalisation épistolaire car elle se justifie seulement par les thèmes rencontrés alors que le domaine public peut également faire grand usage du secret, notamment sous la forme politique du secret d'État. Pour la lettre de Medea à Protheselaüs par exemple, l'intervention de Brutus néglige complètement la relation amoureuse des protagonistes, qui n'est pas sa cible première. L'aide politique et guerrière que Medea, reine de Crète, est susceptible d'apporter au banni Protheselaüs est au contraire déterminante, et formait d'ailleurs l'objectif principal de la lettre. Aussi, si la relation détruite est bien une relation privée, c'est son actualisation ponctuelle sous une forme politique et publique par une lettre secrète qui est visée ${ }^{17}$. Le même jeu subtilement politique sur les interférences entre les domaines du privé et du public se retrouve dans le Roman de Silence. Le recours à la lettre et à une exécution indirecte s'y justifie selon Ebain par la nécessité politique du secret sur le viol dont la reine prétend avoir été victime: par le statut des personnages impliqués dans cette affaire, celle-ci se transforme donc en affaire d'État, et c'est ce statut seul qui justifie qu'elle soit tenue secrète. La même intrication du personnel et du public due au rang des personnages se retrouve enfin dans les lettres substituées de La Manekine et du Roman du comte d'Anjou où elle est rendue manifeste par la double voie empruntée par les nouvelles de la naissance de l'enfant : dans la mesure où celui-ci n'est pas

\footnotetext{
${ }^{17} \mathrm{Si}$ la lettre est secrète en effet, la prise de position ouverte de Medea dans le conflit que cette missive prépare équivaudra nécessairement à une publication de l'amour qu'elle porte à Protheselaüs.
} 
le fils d'un simple particulier, mais d'un grand seigneur de sang royal ${ }^{18}$, c'est moins un nouveau-né qui est annoncé qu'un héritier, et cette nouvelle, officiellement transmise au père par voie épistolaire, est également colportée par la rumeur. Cette diffusion publique parallèle à la diffusion épistolaire pose donc bien le problème : est-ce le secret qui rend la lettre personnelle et donne à sa lecture par un tiers le caractère d'une intrusion, ou bien le transfert de la nouvelle dans le domaine privé ? Ici, il semble en fait que les deux explications doivent être rejetées aussi bien l'une que l'autre : dans la mesure où la nouvelle est par ailleurs colportée, si la lettre cherchait à la rendre secrète, ce serait un secret de Polichinelle, mais ce n'est pas non plus un transfert sur le plan privé auquel elle se livre, puisque ce qui distingue la lettre scellée de la rumeur est justement sa valeur de publication officielle ${ }^{19}$.

\section{Politiques épistolaires du secret : du silence au mensonge}

Pour apporter une solution provisoire au problème, il convient de remarquer que, si la lettre n'est pas plus systématiquement associée au secret qu'elle ne l'est au privé, elle présente en revanche un certain nombre de points communs avec le secret, dont elle semble partager la dynamique de dépassement des frontières établies entre le privé et le public. La première ressemblance tient au polymorphisme dont ils font tous deux preuve. De même que la lettre se prête aux sujets les plus variés, des mesures politiques

18 PhilipPe DE REMI, sire de BEAUMANOIR, La Manekine, dans CEuvres complètes, éd. Hermann SuchIER, Paris, vol. 1, SATF, 1884 ; JEAN MAILlART, Le Roman du comte d'Anjou, éd. Mario RoQues, Paris, CFMA, 1931. Le mari de La Manekine est lui-même roi d'Écosse, quant au comte de Bourges qu'épouse la fille du comte d'Anjou, il est lui-même de lignée royale, comme sa tante le rappelle à plusieurs reprises.

${ }^{19}$ Cette valeur, due à l'apposition du sceau, montre en fait que la valeur officielle de la correspondance repose sur la personnalisation de la communication et l'identification des correspondants. Voir pour une analyse plus détaillée, OUDIN, Fanny, «La pratique épistolaire médiévale entre norme et liberté », Camenulae 2 (juin 2008). 
aux aveux amoureux en passant par les développements didactiques, de même tout sujet est-il susceptible d'être soumis au secret, car c'est le partage du savoir relatif à l'objet qui fait le secret, et non l'objet lui-même. Aussi bien, les sèmes majeurs proposés par Arnaud Lévy ${ }^{20}$ pour définir le secret correspondent également à des traits caractéristiques de la pratique épistolaire. L'association des sèmes /distinction/ et /séparation/, /savoir/ et /autrui/ ou /relation à autrui/ pourrait être reprise telle quelle pour définir la pratique épistolaire. En effet, celle-ci a pour enjeu fondamental une relation à autrui fondée sur la distinction d'un destinataire privilégié qui exclut les autres interlocuteurs potentiels, et articulée autour de la transmission d'une information, c'est-à-dire d'une forme de savoir' ${ }^{21}$.

Par définition, la pratique épistolaire serait donc une pratique du secret, qui devient effectivement, plus que celui de privé, le concept pertinent pour penser la personnalisation à laquelle se livre le geste épistolaire. Avant même de définir les liens que celui-ci établit entre le public et le privé, par rapport auxquels la lettre apparaît comme un texte à double face, à la fois subjectif, personnel, et fortement socialisé, il est donc nécessaire de revenir sur la façon dont le secret participe à la mise en place de la relation et de la communication

${ }^{20}$ LEVY, Arnaud, «Évaluation étymologique et sémantique du mot "secret" », Nouvelle revue de Psychanalyse, 14 (1976), p. 117-129. L'auteur propose de définir le secret comme une structuration des relations sociales autour d'une pratique de la dissimulation qui induit d'une part un rapport de domination pensé en termes d'inclusion ou d'exclusion et d'autre part un consensus de paix nécessaire à l'illusion de l'existence collective.

${ }^{21}$ Cette association de la lettre au savoir est récurrente : il existe toute une tradition de lettres didactiques qui forme un sous-genre au sein de la pratique épistolaire du Moyen Âge aussi bien en latin qu'en langues vulgaires, au point que ce didactisme a pu être pensé comme un trait distinctif des épîtres médiévales par rapport à celles de la Renaissance. De plus, il est significatif que dans la lettre aux trois dons d'Eracle le savoir que la lettre confère au personnage soit pensé en termes de dissimulation et de révélation des tares ou des qualités cachées des êtres, des animaux et des choses (v. 229-278). 
épistolaires : elle connaît plusieurs degrés, de la simple dissimulation au mensonge.

La forme la plus simple du secret reste le silence : peut-être explique-telle les lectures silencieuses des lettres, très étranges par rapport à ce qui est connu des pratiques de lecture courantes au Moyen Âge. Ce n'est pas vraiment pour les secrets amoureux que la lecture se fait silencieuse, mais plutôt pour des secrets politiques qui ont trait, le plus souvent, aux lettres substituées. Que ce soit dans le Roman du comte d'Anjou, dans celui de Silence ou dans La Manekine, la remise publique de la lettre devant une cour nombreuse donne d'abord lieu à une lecture à voix basse sur place, puis à un mouvement de retraite vers une pièce plus isolée. Dans Silence, le roi de France réunit un conseil privé avant de retourner auprès de ses barons ; dans les deux autres romans, le comte de Bourges et le roi d'Écosse se retirent dans une chambre pour donner libre cours à leurs sentiments. Le passage vers une pièce qui relève du domaine privé répond à la nécessité d'une dissimulation et à la volonté de sauvegarder les apparences: du comte, il est précisé que « mains qu'i puet en fet semblant» (v. 3546), du roi d'Écosse que

«Et pour chou que apercevoir

Ne s'en puissent la gent estrange,

De la u il ert son lieu cange :

Lui tierch en sa cambre est venus. ${ }^{22}$

La volonté de dissimulation et le rapport à autrui qui la justifie sont ici explicites et sont encore précisés aux vers 3283-3286 par le discours du chevalier qui joue d'une rime significative entre « reveler » et « celer»:

«Ne ne voelliés ja reveler

Ceste novele mais celer ;

${ }^{22}$ PhilipPe De Remi, sire de BeAumanoir, La Manekine, v. 3218-3221. 
Car il fait mauvais tenir conte

De ce dont on puet avoir honte. »

Pour suspendre l'effet d'une lettre porteuse d'une information néfaste, il faut contrer son ébruitement, car seule la diffusion de l'information lui confère sa pleine puissance en la rendant publique et en l'officialisant. Ce jeu de rimes rend sensible la double dynamique qui préside à tout secret, ainsi qu'à sa perception éthique. Ce n'est plus la transparence du secret révélé qui est évaluée positivement, comme cela se produit ensuite pour la dissipation des quiproquos, mais au contraire l'opacité de l'écrit qui est vantée comme garante et d'un secret d'État et d'une protection de la vie privée.

Le secret n'est pas cantonné dans la pratique épistolaire à la seule lecture, où il n'agit qu'a posteriori sur la diffusion du contenu de la missive : il peut aussi être partie prenante de la conception du message et ainsi s'intégrer à la dynamique même de la communication. Deux configurations principales sont possibles : ou bien la dissimulation reste partielle et touche aux raisons de l'envoi, qui sont ignorées du public voire du messager, ou bien il s'agit de dissimuler l'acte de communication lui-même, qui est effectué secrètement.

C'est à ce second cas de figure que correspond l'envoi par Sornegur d'un clerc porteur d'une missive offrant au roi de France de régler l'issue de la guerre par un combat singulier ${ }^{23}$. La dissimulation est alors rendue patente par le contraste entre l'insignifiance du messager unique, simple clerc et confident privé de Sornegur, et le faste de la cour de France, où Lohiers reçoit cet émissaire entouré des plus hauts dignitaires de la hiérarchie ecclésiastique : tous ces spectateurs, quoique muets, ne peuvent qu'écraser de leur poids la

\footnotetext{
${ }^{23}$ Partonopeus de Blois, éd. Olivier Collet et Pierre-Marie Joris, Paris, Livre de Poche, «Lettres gothiques », 2005, v. 2703 sqq.
} 
simplicité du personnage qui s'est présenté devant eux. Le même contraste se retrouve, à l'autre bout de la chaîne de communication, dans le compte-rendu de cet échange fait par chacun des deux rois à son conseil. Alors que Lohiers recourt à la solennité d'une parole directe, à haute voix, devant la cour au grand complet, et fait suivre cette séance de cérémonies religieuses collectives, Sornegur fait part de sa décision à un conseil restreint qui s'en retourne chez lui en silence. De plus, il fait passer cette révélation par le truchement de la lecture, toujours silencieuse, de la lettre de Lohiers, et non par sa propre parole: tout se passe donc comme si, pour suppléer à la défaillance d'autorité de sa propre parole, il devait recourir à celle de son ennemi. Ces contrastes pointent le caractère anormal de ce secret dû à une véritable crise du pouvoir politique au sein de l'armée païenne, ébranlé par les manœuvres du traître Marès: il reste important de noter que la missive apparaît alors comme le recours privilégié pour contourner le conseil public et résoudre le problème en recourant à l'intervention d'un tiers.

C'est également la résolution d'une crise par l'intervention d'un tiers qui justifie chez Ebain le recours à la lettre pour contourner le problème en détournant sa résolution. Mais il introduit une dimension supplémentaire en transformant le silence en mensonge : dans les discours qu'il adresse à la reine pour justifier son geste, le recours à la lettre est en effet introduit par une rime entre «songe» et «mensonge» (v. 4245-4246). Par opposition avec le châtiment immédiat, direct et irréfléchi qu'elle lui recommande, la préservation du secret repose sur l'introduction d'une distance et d'une médiation dans l'exercice de la justice, c'est-à-dire sur le geste épistolaire médiateur. Celui-ci se trouve donc inscrit dans une politique et une poétique du silence conçu comme une ruse qui le transforme en mensonge en jouant de l'illusion (le «songe»). Ce n'est donc pas un hasard si les rimes suivantes 
entre «brief » et « cief » (à couper) ou «fief » (à donner) traduisent toutes silencieusement et obliquement la duplicité d'intention d'Ebain qui donne à son épouse l'illusion qu'il va tromper Silence pour mieux la tromper elle et détourner sa soif de vengeance. La dissimulation apparaît ainsi comme le fondement premier et indispensable du mensonge qui permet de jouer des apparences introduisant des biais dans la communication: ce n'est plus seulement le silence (la vérité, rien que la vérité, mais non toute la vérité), mais l'illusion mensongère qui participe à l'élaboration de toute communication et de toute relation sociale. Or ces trois éléments étroitement associés par le roman ${ }^{24}$ sont aussi, au moins pour les deux derniers, fondateurs du geste épistolaire, qui repose sur la représentation virtuelle, l'illusion de présence et sur la médiation. Cette configuration confirmerait donc la parenté profonde de la lettre avec le secret plutôt qu'avec le privé.

Le secret épistolaire, garanti par le sceau apposé au bas de la lettre, permet de comprendre de quels procédés joue la personnalisation de la communication dans et par la relation épistolaire : elle se définit en propre par l'individualisation d'un dialogue, et non d'un simple locuteur. Les scènes de transgression du secret le montrent clairement, puisque c'est la transmission de la lettre qui fait l'objet d'une effraction et que c'est toujours l'ensemble de la relation qui s'en trouve affectée. Par rapport aux autres formes de poésie personnelle, la poésie épistolaire se singularise donc par la mise en œuvre d'une intersubjectivité et non d'une simple subjectivité. La privatisation de l'échange ne vaut pas repli sur la sphère de l'intime, car la parole reste

${ }^{24}$ Il faut remarquer que le même approfondissement du secret en ruse qui tourne au mensonge se situe à l'origine de l'intrigue, puisque c'est l'ambiguïté androgyne du nom de Silence qui permet de dissimuler sa nature et finalement de mentir en la faisant passer pour ce qu'elle n'est pas : la réflexion sur les rapports entre le secret et le mensonge, fondateurs de la communication, semble donc bien s'inscrire au frontispice de l'œuvre. 
orientée vers la construction d'un lien social dans et par une écriture individualisée qui en retour nourrit sa propre identité des codes sociaux dont elle joue. La pratique épistolaire ne se contente donc pas de mettre à jour une tension entre les sphères du public et du privé en se jouant de leurs frontières : elle les articule véritablement en faisant reposer la valeur officielle du texte sur l'individualisation de l'écrit, c'est-à-dire sur son assomption par un locuteur à l'intention d'un autre. Révélatrice est de ce point de vue la figure du tiers qui reste toujours associée au secret épistolaire : qu'il apparaisse comme un médiateur appelé par la correspondance ou au contraire comme un obstacle à la communication, il rappelle toujours que la correspondance est une pratique à la fois interpersonnelle et médiatisée, oblique, donc susceptible de se transformer en véritable polyphonie. 